\title{
Three-dimensional multiresonant lossy sonic crystal for broadband acoustic attenuation: Application to train noise reduction
}

\author{
T. Cavalieri ${ }^{\mathrm{a}}$, A. Cebrecos ${ }^{\mathrm{a}}$, J.-P. Groby ${ }^{\mathrm{a}}$, C. Chaufour ${ }^{\mathrm{b}}$, V. Romero-García ${ }^{\mathrm{a}}$ \\ ${ }^{a}$ Laboratoire d'Acoustique de l'Université du Mans, LAUM, UMR CNRS 6613, Av. Olivier \\ Messiaen, Le Mans, France \\ ${ }^{b} S N C F$, Direction Innovation $\&$ Recherche, Av. des Terroirs de France, Paris, France
}

\begin{abstract}
Broadband acoustic attenuation produced by a three dimensional (3D) locally resonant sonic crystal (LRSC), exploiting both the multiple coupled resonances and the Bragg band gaps, is numerically and experimentally reported in this work. The LRSC is made of square cross-section scatterers arranged on a square lattice and periodically incorporating both quarter-wavelength and Helmholtz resonators along their heights. Local resonators of different types are combined with the periodicity of the system generating multiple coupled resonances at low frequencies and opening Bragg band gaps respectively. This twofold coupling produces a strong broadband attenuation: a large insertion loss (IL), with an average value of $16.8 \mathrm{~dB}$, covering three and a half octaves from $350 \mathrm{~Hz}$ to 5000 $\mathrm{Hz}$ with a LRSC of $30 \mathrm{~cm}$ width. This frequency band corresponds to one of the several railway noise sources (rolling noise, traction auxiliaries, etc.). A simplified 2D LRSC is finally analyzed numerically in a real train-track configuration, showing the efficiency of the proposed design to attenuate the railway rolling noise.
\end{abstract}

Keywords: sonic crystals, acoustic barrier, acoustic metamaterial, resonators

\section{Introduction}

The acoustic properties of locally resonant sonic crystals (LRSC) [1, 2, 3, 4] have been extensively exploited over the last years for applications in acoustics based on acoustic filters [5], lenses [6], waveguides [7, energy trapping systems 5 [8] and isolation devices [9], among others. The design of acoustic barriers for environmental noise remains yet the most widespread application [9, 10, 11, 32, 13, 14, 15, 16, 17, 18, 19, 20, 21. LRSC are effectively excellent candidates in a plethora of applications to efficiently attenuate airborne sound thanks to their broadband filtering ability. This ability yields in the coupling of both the 10 periodicity (band gaps) and the coupled local resonances (stop bands), 9, 32, [13, 21. Moreover, air and light might flow through LRSC, which is particularly suitable in urban contexts. LRSC effectively exhibit a drastic reduction of air 
flow resistivity when compared to usual sound barriers, which directly implies a reduction of the foundation costs [20].

15 Environmental noise includes railway noise, which encompasses different kind of noise sources. Railway noise is generally classified in three categories: auxiliary, rolling, and aerodynamic noises. Auxiliary noise arises from the different technical equipments, like diesel engine, power transformer, converter, etc. These equipments are located in the upper or lower parts of locomotives and

20 coaches, either on the roof or at the wheel height. This noise is predominant during parking periods and at very low train speeds $\left(\leq 50 \mathrm{~km} \cdot \mathrm{h}^{-1}\right)$. Rolling noise is radiated by the rolling stock and the track, due to the vibration originated at the wheel-track contact, mostly because of rail roughness and corrugation. The global noise radiation is therefore the sum of different contributions arising

25 from the train wheels, the rail and the sleepers of the track. This second type of noise is predominant for train speeds between 80 and $300 \mathrm{~km} \cdot \mathrm{h}^{-1}$. Finally, the aerodynamic noise appears for higher train speeds, up to $300 \mathrm{~km} \cdot \mathrm{h}^{-1}$.

In this article, we focus on rolling noise and the design of a LRSC in the corresponding frequency range. The attenuating properties of a 3D LRSC made

30 of square cross-section scatterers arranged on a square lattice and periodically incorporating both quarter-wavelength resonators (QWR) and Helmholtz resonators (HR) along their heights are theoretically and experimentally studied. Viscothermal losses are accounted for both in the QWR and HR by using the Zwikker and Kosten formulae 22, 23. The combination of stop bands, due to

35 local resonant scatterers generating multiple coupled resonances at low frequencies, with bandgaps, due to periodicity, is exploited to produce the broadest and largest possible value of the insertion loss $(I L)$. The HR are deep subwavelength resonators, the resonance of which is in our case $\lambda / l=18$, where $\lambda$ is the wavelength and $l$ is the length of the HR. The QWR are designed such

40 that their resonance frequency coincides with the Bragg band gap. Numerical predictions and experimental results show a strong broadband attenuation, the LRSC exhibits a large $I L$ covering three and a half octaves with an average value of $16.8 \mathrm{~dB}$ from $350 \mathrm{~Hz}$ to $5000 \mathrm{~Hz}$. Finally, a simplified 2D LRSC is numerically analyzed in a real train-track configuration, showing the efficiency 45 of the proposed design for the attenuation of rolling noise.

The article is organized as follows. In Section 2 the modeling of both the local resonators and the LRSC is presented. The way the viscothermal losses are accounted for in the local resonators and the conditions used to calculate the dispersion relation and the scattering problem of the 3D LRSC are explicitly ${ }_{50}$ presented. Section 3 shows the experimental setup, while the results are discussed in Section 4, also including a simplified 2D LRSC numerically analyzed in a real train-track configuration. Finally, concluding remarks are summarized in Section 5 .

\section{Modeling}

The modeling of the local resonators as well as the whole periodic system, including the scatterers together with the local resonators, are detailed in the 
following subsections.

\subsection{Local lossy resonators}

Figures 1(a) and (b) show the schematics of the QWRs and HRs, respectively. The QWR consists of a cylindrical borehole carved out of a square-rod scatterer. The HR is composed of a cylindrical neck added to the same cylindrical borehole. The propagation of acoustic plane waves in a circular cross-section duct of radius $r_{c}$ accounting for the viscothermal losses is described by the complex and frequency dependent effective density and bulk modulus, as described in Ref. 23

$$
\begin{gathered}
\tilde{\rho}_{c}=\rho_{0}\left(1-\frac{2 J_{1}\left(r_{c} \tilde{G}_{r}\right)}{r_{c} \tilde{G}_{r} J_{0}\left(r_{c} \tilde{G}_{r}\right)}\right)^{-1}, \\
\tilde{\kappa}_{c}=K_{0}\left(1+(\gamma-1) \frac{2 J_{1}\left(r_{c} \tilde{G}_{k}\right)}{r_{c} \tilde{G}_{k} J_{0}\left(r_{c} \tilde{G}_{k}\right)}\right)^{-1},
\end{gathered}
$$

where $\tilde{G}_{r}=\sqrt{\imath \omega \rho_{0} / \eta}$ and $\tilde{G}_{k}=\sqrt{\imath \omega \operatorname{Pr} \rho_{0} / \eta}$, with $\imath=\sqrt{-1}, \rho_{0}$ is the density, $K_{0}=\gamma P_{0}$ is the bulk modulus of air, $\gamma$ is the specific heat ratio, $P_{0}$ is the atmospheric pressure, $\operatorname{Pr}$ is the Prandtl number and $\eta$ is the dynamic viscosity. We notice that the frequency dependent effective properties of more complex geometries have been also obtained in Ref. [23. Alternatively, we can define the effective impedance $\tilde{Z}_{c}=\tilde{\rho}_{c} \tilde{\kappa}_{c}$ and wavenumber $\tilde{k}_{c}=\omega \sqrt{\tilde{\rho}_{c} / \tilde{\kappa}_{c}}$ from Eqs. (1) and $(2)$.

Using the effective parameters for a cavity element (Eqs. (112)), the impedance of a QWR made of a circular borehole of diameter $d_{q w r}=2 r_{q w r}$ (where $r_{q w r}$ is the radius of the borehole) and length $l_{q w r}$ can be written as [24]

$$
\tilde{Z}_{\mathrm{QWR}}=-\imath Z_{q w r} \cot \left(k_{q w r} l_{q w r}\right),
$$

where $Z_{q w r}$ and $k_{q w r}$ are obtained from Eqs. (1.2).

It is worth noting here that this expression is not exact as long as correction length due to radiation is not included. The characteristic impedance accounting for the borehole radiation can be expressed as [25]:

$$
\tilde{Z}_{\mathrm{QWR}}=-\imath Z_{q w r} \cot \left(k_{q w r} l_{q w r}\right)+\imath k_{q w r} \Delta l Z_{q w r},
$$

where the correction length reads as $\Delta l=0.6 r_{c}$.

Similarly, the impedance of a HR made of a cylindrical neck of diameter $d_{n}=2 r_{n}$ and length $l_{n}$, and a cylindrical cavity of diameter $d_{c}=2 r_{c}$ and length $l_{c}$ can be written as 26 .

$$
\tilde{Z}_{\mathrm{HR}}=\imath Z_{n} \frac{A-\tan k_{n} l_{n} \tan k_{c} l_{c}}{A \tan k_{n} l_{n}+\tan k_{c} l_{c}}
$$




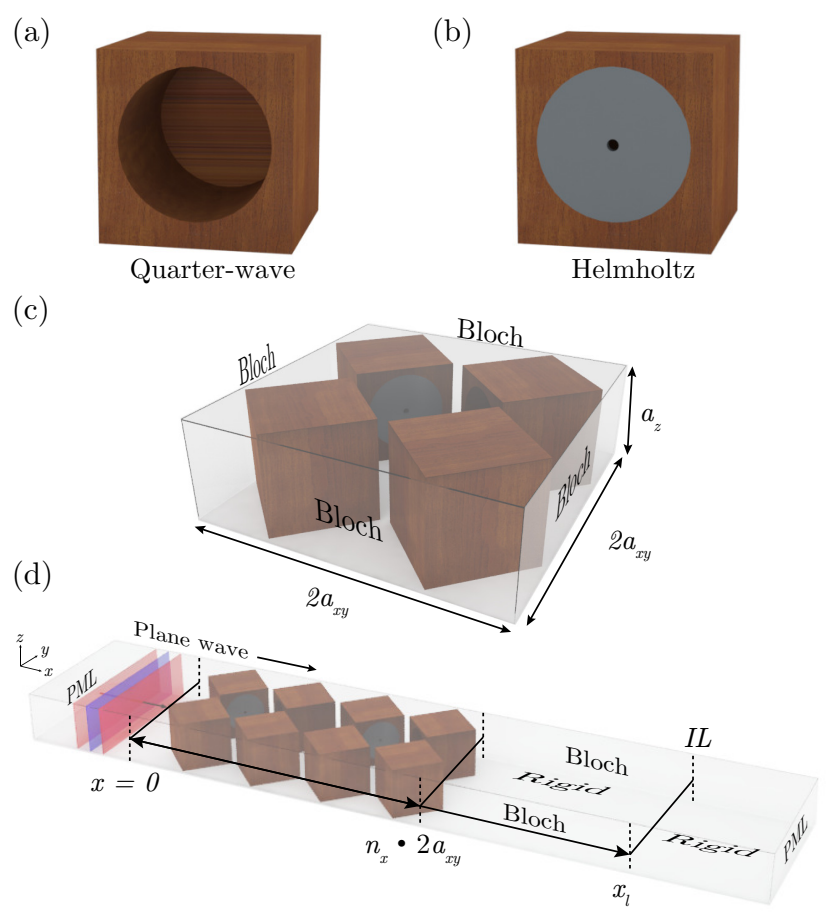

Figure 1: Schematics of the modeling. Local resonators: (a) Quarter-wavelength and (b) Helmholtz resonators. (c) Three-dimensional unit cell employed for the eigenvalue problem. (d) Scattering problem for the calculation of the IL of an infinite LRSC slab.

where $A=Z_{c} / Z_{n}$ and $k_{n}\left(k_{c}\right), Z_{n}\left(Z_{c}\right)$ are the effective wavenumbers and 85 effective characteristic impedances in the neck (cavity) of the HR.

It is again worth noting here that this expression should account for the radiation of each element. Therefore, the correct expression of the characteristic impedance becomes [26]:

$$
\tilde{Z}_{\mathrm{HR}}=-\imath \frac{c_{n} c_{c}-Z_{n} k_{n} \Delta l c_{n} s_{c} / Z_{c}-Z_{n} s_{n} s_{c} / Z_{c}}{s_{n} c_{c} / Z_{n}-k_{n} \Delta l s_{n} s_{c} / Z_{c}+c_{n} s_{c} / Z_{c}}
$$

where $c_{n}=\cos \left(k_{n} l_{n}\right), c_{c}=\cos \left(k_{c} l_{c}\right), s_{n}=\sin \left(k_{n} l_{n}\right), s_{c}=\sin \left(k_{c} l_{c}\right)$. The 90 length correction is deduced from the addition of two correction lengths $\Delta l=$ $\Delta l_{1}+\Delta l_{2}$ respectively defined as $\Delta l_{1}=0.82\left[1-1.35 \frac{r_{n}}{r_{c}}+0.31\left(\frac{r_{n}}{r_{c}}\right)^{3}\right] r_{n}$ and $\Delta l_{2}=0.6 r_{n}$

The first length correction, $\Delta l_{1}$, is due to pressure radiation at the discontinuity from the neck to the cavity of the HR [27, while the second length 95 correction, $\Delta l_{2}$, comes from the radiation at the discontinuity from the neck to the surrounding medium [28]. 


\subsection{Numerical modeling of the periodic system}

We introduce now the numerical models of the LRSC utilized in this work, which are solved using the Finite Element Method (FEM). We solve first an

100 LRSC, and then a scattering problem using two different LRSC slabs, one having an infinite transversal length and the other one a finite length by considering the viscothermal losses in the resonators but not outside the scatterers. The former represents a case close to a real acoustic barrier of very large transversal the experimental results measured in an anechoic chanber. For all three case the spatial domain is discretized creating at least 6 elements per wavelength for the highest analyzed frequency, $\lambda_{\min }\left(6000 \mathrm{~Hz}\right.$, hence $\left.\lambda_{\min }=5.7 \mathrm{~cm}\right)$.

\subsubsection{Eigenvalue problem: dispersion relation}

110

The 3D unit cell of the LRSC used in this study is shown in Fig. 1(c). It is composed of four scatterers embedding resonators of different type and rotated 45 degrees around their vertical axes with respect to the lattice orientation. All four resonators are facing the center of the unit cell in such a way that identical resonators are facing one with each other, i.e., the two HRs and the two from the fact that the broadest achievable band gap of a two-dimensional sonic crystal composed of square cross-section scatterers is produced when these are rotated 45 degrees around their axes [29]. In addition, the selected distribution of different types of resonators is obtained from the analysis of different configAppendix A

The unit cell shown in Fig. 1(c) is discretized using $4.5 \times 10^{4}$ elements. The boundaries of the wooden square cross-section scatterers are considered acoustically rigid and Floquet-Bloch boundary conditions are assumed on the edges of the unit cell to account for the periodicity of the system (see Fig. 1(c))

$$
p(\vec{r}+\vec{R})=p(\vec{r}) e^{\imath \vec{k}_{B} \cdot \vec{R}},
$$

where $\vec{R}$ is the lattice vector and $\overrightarrow{k_{B}}$ is the Bloch vector. By fixing the wavevector, $\overrightarrow{k_{B}}=\left(k_{B x}, k_{B y}, k_{B z}\right)$ at a specific location along the path of the irreducible Brillouin zone, the corresponding eigenfrequencies are evaluated, and the dispersion relation of the periodic structure is calculated. In the present case, the (3) to the particular distribution of different resonators in the unit cell. Hence, the main directions of symmetry in the reciprocal space are following the path $\Gamma \mathrm{X}-\mathrm{XM}-\mathrm{M} \Gamma-\Gamma \mathrm{M}^{\prime}$, i.e., considering the ranges $k_{B x}=\left[-\pi / 2 a_{x y}, \pi / 2 a_{x y}\right]$ and $k_{B y}=\left[0, \pi / 2 a_{x y}\right]$, for $k_{B z}=0$.

\subsubsection{Scattering problem: infinite LRSC slab}

The geometry of the scattering problem using an infinite LRSC slab, i.e. a finite thickness and infinite width system, as it is shown in Fig. 1(d). In the 
illustrated example, the infinite slab is composed of $n_{x}=2$ unit cells along the propagation direction ( $x$-direction, thickness); boundary conditions along the $y$ - and $z$-directions are chosen such that the structure can be considered periodic along these two directions. A plane wave impinges the structure from the negative $x$-axis ( $\Gamma \mathrm{X}$ direction). Perfectly matched layers (PML) are applied on both ends along the $x$-direction. The whole solution domain is discretized into $2.2 \times 10^{5}$ elements.

145 The pressure field is evaluated in the axial plane, i.e., the $x y$-plane, for $z=a_{z} / 2$. In order to evaluate the attenuation of the 3D LRSC, the $I L$ at frequency $f$ is spatially averaged using $M$ points along a line ranging from $y=\left[-a_{x y} / 2, a_{x y} / 2\right]$ behind the LRSC at $x=x_{l}=0.65 \mathrm{~m}$ (see Fig. 1(d)).

$$
\langle I L(f)\rangle=20 \log _{10}\left(\frac{\sum_{i=1}^{M}\left|\tilde{p}_{r e f}\left(\vec{r}_{i}, f\right)\right|}{\sum_{i=1}^{M}\left|\tilde{p}_{s c}\left(\vec{r}_{i}, f\right)\right|}\right) .
$$

where $\left|\tilde{p}_{r e f}(\vec{r}, f)\right|$ and $\left|\tilde{p}_{s c}(\vec{r}, f)\right|$ are the absolute values of the acoustic pressure in the absence and in the presence of the 3D LRSC, respectively. It is worth noting here that the length $x_{l}$ is large enough to avoid the contribution from evanescent waves scattered by the structure.

\subsubsection{Scattering problem: finite LRSC slab}

The finite slab is a LRSC with finite thickness and width. In our case we choose $n_{x}=2$ unit cells along the $x$ direction (thickness), $n_{y}=9$ unit cells along the $y$ direction (width) and of infinite height along the $z$ direction is considered and will be presented later in Section 4. The acoustic source is modeled as a piston of infinite length along the $z$ direction placed at $1.4 \mathrm{~m}$ from the structure. Boundary conditions along the $z$-direction are chosen such that the LRSC can

160 be considered of infinite height. PML layers are considered on both ends on $x$ and $y$-directions, hence the only contributions from the incident field behind the LRSC are those propagating through the structure and from its lateral edges. The spatial discretization is performed using $1.8510^{6}$ elements.

\section{Experimental setup}

Our attention is now turned into the description of the experimental setup, introducing the geometry of the locally resonant scatterers, including both types of resonators; the whole periodic system, i.e., the finite LRSC slab and the instrumentation and experimental methods employed for the measurements.

\subsection{Locally resonant scatterers}

170

The scatterers consist of wooden rods of square cross-section. Each rod is $0.05 \times 0.05 \times 2.00 \mathrm{~m}^{3}$ (i.e., $L=0.05 \mathrm{~m}$ ) and incorporates 29 resonators in its central area along the $z$-direction separated by a distance $a_{z}=0.05 \mathrm{~m}$. Each QWR has a diameter $d_{Q}=0.035 \mathrm{~m}$ and depth $l_{Q}=0.04 \mathrm{~m}$ (see Fig. 2(a)). HRs are obtained by inserting a $l_{n}$-thick PVC annular disc on top of the QWRs. 
175 The inner hole of each PVC annular disc plays the role of the HR neck. The neck diameter is $d_{n}=0.004 \mathrm{~m}$ and its length is $l_{n}=0.004 \mathrm{~m}$. The diameter of the HR cavity is $d_{c}=0.035 \mathrm{~m}$ and its depth $l_{c}=0.036 \mathrm{~m}$ (see Fig. 2(b)).

(a)

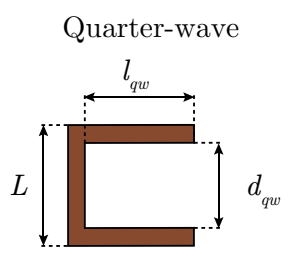

(c)

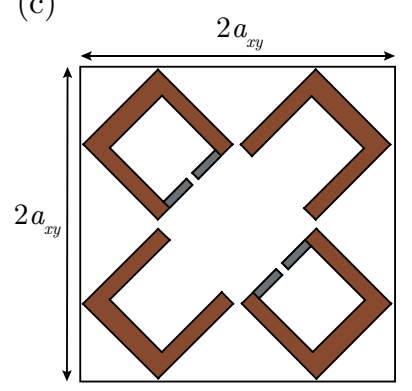

(e) (b)

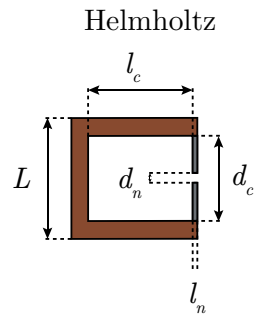

(d)

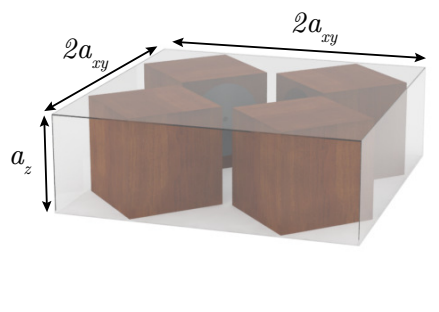

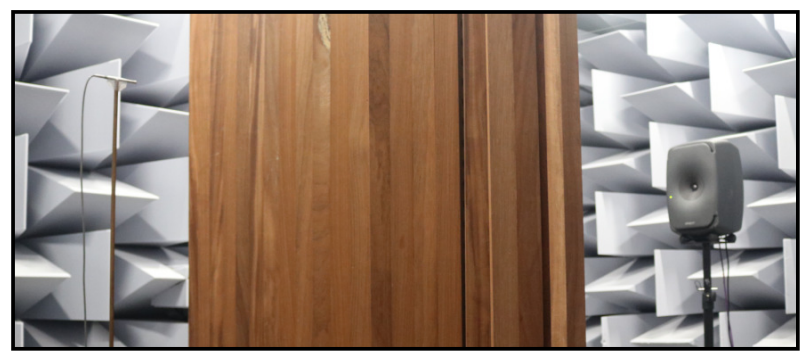

Figure 2: Experimental set-up. (a), (b) Transversal views of both types of resonators, QWR and HR, respectively. (c), (d) Schematic diagram of the unit cell and main geometrical parameters. (e) Picture of the experimental set-up in the anechoic chamber.

\subsection{Periodic arrangement of locally resonant scatterers: LRSC}

The LRSC is composed of an arrangement of locally resonant scatterers, distributed in a $2 \mathrm{D}$ square lattice with a lattice period $a_{x y}=0.075 \mathrm{~m}$, as shown in Fig. 2(c). The vertical lattice period of the resonators along the scatterer height is $a_{z}=0.050 \mathrm{~m}$. Therefore, the $3 \mathrm{D}$ unit cell is a parallelepiped rectangle with a lattice constant $2 a_{x y}$, as shown in Fig. 2 (d). The LRSC experimentally analyzed is built by using 2 unit cells along the propagation direction, $x$-direction, and 9 unit cells along the transverse direction, $y$-direction. The filling fraction of the LRSC, defined as the ratio of the volume of the scatterers $V_{s c}$ over the 
total volume of the unit cell $V_{\text {cell }}$, is $f f=\frac{V_{s c}}{V_{\text {cell }}} \approx 0.314$. $V_{s c}$ is defined in this work as the solid part of the unit cell.

Experimental measurements are performed using a microphone B\&K 1/4" type 4135 . The acoustic source is a loudspeaker Genelec 8351A. The excitation signal is a sweep sine function ranging from 50 to $6000 \mathrm{~Hz}$ with a step of $12 \mathrm{~Hz}$. The positions of the microphone along the measurement line are controlled by a Zaber LSQ 1D robotized arm. The acquisition of the acoustic signal is performed using a Stanford SR 785 spectrum analyzer. A picture of the experimental setup

195 is represented in Fig. 2(e). The loudspeaker used to generate the acoustic field in the anechoic chamber is placed at an approximate distance of $1.4 \mathrm{~m}$ from the LRSC. A single microphone is utilized to measure the transfer functions between the electrical and the acoustic signal measured at every point along the line behind the LRSC. Only propagation along the $x-y$ plane is considered.

\subsection{Insertion Loss}

The attenuation of the LRSC is evaluated measuring the $I L$ along a line behind the sample at $x_{l}=0.65 \mathrm{~m},(x=0$ is located following the same convention presented in Fig. 11(d)). This measurement line spans the interval $y_{l}=[-0.225,0.225] \mathrm{cm}$ with $1 \mathrm{~cm}$ step $(M=46$ points). The transfer function between the signal registered by the microphone and the input electrical signal is measured twice along this line, i.e., in the absence and in the presence of the LRSC, and later spatially averaged. The absolute value of the spatially averaged complex transfer function at point $\vec{r}_{i}$ and frequency $f$ is given by

$$
\langle|\tilde{H}(f)|\rangle=20 \log _{10}\left(\frac{1}{M|\tilde{V}(f)|} \sum_{i=1}^{M}\left|\tilde{p}\left(\vec{r}_{i}, f\right)\right|\right),
$$

where $\tilde{H}=\left(\tilde{H}_{r e f}, \tilde{H}_{s c}\right)$, and $\tilde{p}=\left(\tilde{p}_{r e f}, \tilde{p}_{s c}\right)$ is the measured acoustic pressure along the line. Note that subscripts refer to measurements performed in the absence and in the presence of the LRSC. The spatially averaged $\langle I L\rangle$ is obtained making use of Eq. (8).

\section{Results}

\subsection{Characterization of the local lossy resonators}

The local resonators used in the LRSC are characterized in this subsection. A numerical full wave simulation mimicking an impedance tube measurement is performed in order to obtain the acoustic impedance of the local resonators. The configurations of the impedance tube considered for the QWR and HR are shown in Figs. 3(a, b), respectively. To characterize the acoustic properties of 220 the local resonators, the effective properties given by Eqs. (11-2) are introduced in their respective domains. The acoustic impedance of each resonator is recovered from the numerical model evaluating the transfer function, and compared to the analytical expressions given by the Eqs. (4, 6). Note that the previous analytical models have been already validated experimentally [30, 31]. 
Figures $3(\mathrm{c}, \mathrm{d})$ show the real and imaginary part of the normalized impedance of the QWR and HR, respectively. Continuous and dashed lines correspond to analytical predictions from Eqs. (4. 6), while symbols represent results from the numerical model presented previously. Numerical and analytical results are in excellent agreement, which validate the model used in this work to account 230 for the losses. Note that viscothermal losses are considered in both calculations, hence the value of the resonance frequency represents the behavior of the resonator itself, with no coupling to other resonant elements. The resonance frequency corresponds to the frequency at which $\Im\left(\tilde{Z}_{\text {res }}\right)=0$ (where res $=$ QWR, HR). By doing so, the resonance frequency of the HR is found at $f_{H R}=380 \mathrm{~Hz}$ and that of the QWR at $f_{Q W R}=2000 \mathrm{~Hz}$.

(a)

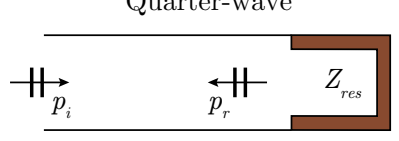

(c)

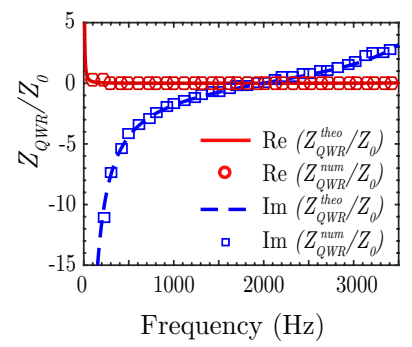

(b)

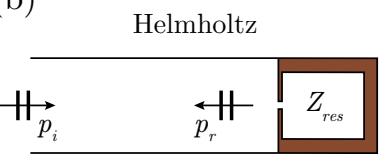

(d)

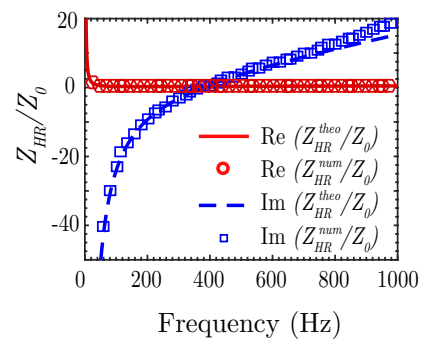

Figure 3: Characterization of the local resonators, comparison between analytical and numerical results. (a), (b) show configurations in an impedance tube with QWR and HR, respectively. Real (red continuous line for analytics and red open circles for numerics) and imaginary (blue dashed line for analytics and blue open squares for numerics) parts of the normalized acoustic impedance of the (c) QWR, $Z_{Q W R} / Z_{0}$ and (d) HR, $Z_{H R} / Z_{0}$.

\subsection{Locally resonant sonic crystal}

\subsubsection{Dispersion relation}

The dispersion relation along the main directions of symmetry (ГХ-ХМ-МГ$\left.\Gamma M^{\prime}\right)$ for the unit cell described in Section 2 is shown in Fig. 4(a). The several

bands shown in the dispersion relation are due to the folding effect related to the supercell behaviour of the structure. At low frequencies (around $400 \mathrm{~Hz}$ ) a full stop band is observed due to the resonance of the HRs. The pressure distribution of the unit cell (eigenvector) at the resonance frequency of the HR corresponding to point $\mathrm{X}$ of the dispersion relation is shown in Fig. 4(b). At this frequency,

lower than the Brago band gaps are opened in the $\Gamma \mathrm{X}$ direction as a consequence of additional cavity mode 
excitation [21]. For example, the pressure distribution at frequency $1190 \mathrm{~Hz}$ at point $\Gamma$ is shown in Fig. 4(c), for which a cavity mode in the external cavities of the unit cell is well activated creating a band gap around this frequency in the $\Gamma \mathrm{X}$ direction. Finally, we show the eigenvector at frequency $1600 \mathrm{~Hz}$ at point $\Gamma$ (see Fig. 4(d)). At this frequency, the coupled QWRs open another stop band, as shown in Fig. 4(e). At higher frequencies, the combined effects of the QWRs and the periodicity produce a full band gap between $1600 \mathrm{~Hz}$ and $2553000 \mathrm{~Hz}$. Higher band gaps can be also observed in the dispersion relation due to periodicity and higher order resonant modes.
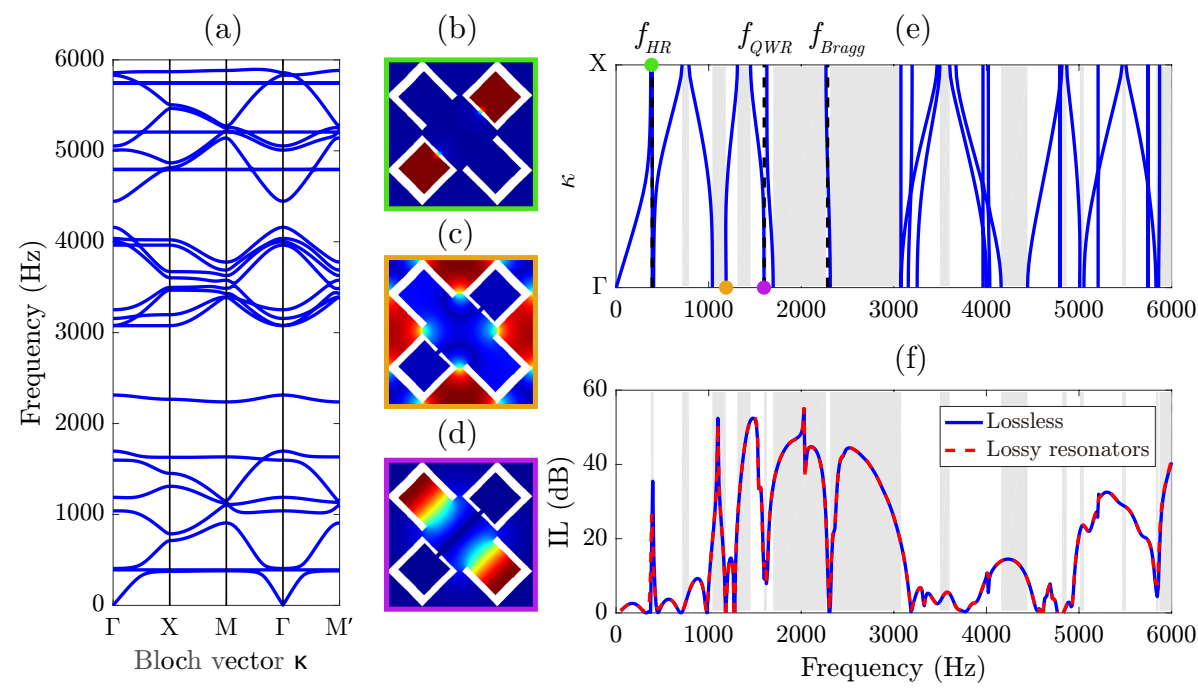

Figure 4: Numerical characterization of the LRSC. (a) Dispersion relation along the main symmetry directions. Eigenvectors at particular frequencies: (b) resonance frequency of the $\mathrm{HR}, f_{H R}=390 \mathrm{~Hz}$, (c) aditional cavity modes at $1190 \mathrm{~Hz}$, (d) resonance frequency of the QWR, $f_{Q W R}=1600 \mathrm{~Hz}$. (e) Dispersion relation along the $\Gamma \mathrm{X}$ direction. (f) Numerical IL for an infinite LRSC slab.

\subsubsection{IL of an infinite LRSC slab}

To analyze in detail the effect of the HRs, the QWRs and periodicity, the dispersion relation along the $\Gamma \mathrm{X}$ direction shown in Fig. 4(e) is compared to the $I L$ produced by an infinite LRSC slab composed of $n_{x}=2$ unit cells. The $I L$ produced by this infinite slab is shown in Fig. $4(\mathrm{f})$ for the lossless (blue continuous line) and the lossy (red dashed line) cases. The attenuation peaks observed in the $I L$ spectrum are in very good agreement with the band gaps obtained from the eigenvalue problem. At the resonant frequency of the HR, $f_{H R}=390 \mathrm{~Hz}$, we observe an $I L$ peak of amplitude $35 \mathrm{~dB}$. Interestingly, for higher frequencies the coupling of the QWR and the effect of periodicity produces $I L$ peaks of amplitude $40 \mathrm{~dB}$. Note that the losses are only accounted for in the resonators, therefore their main effects are manifested at their resonance 
frequencies. The influence of losses in the case of the HR is significant, producing an impedance mismatch and therefore a reduction of the $I L$ peak at 390 Hz. For the QWR, the effects of losses are mostly negligible.

The analysis of an infinite LRSC slab is of large importance to have an idea of the effect of a large barrier, as it would be the case for an application in the context of railway noise control. However, in many practical situations, and in 275 particular for this work, the experimental analysis is performed in an anechoic chamber using a structure of finite transversal length. Hence the interference pattern produced by both edges of the structure should be accounted for in the numerical simulations, as shown in the next Section.

\subsubsection{IL of a finite LRSC slab}

(a)

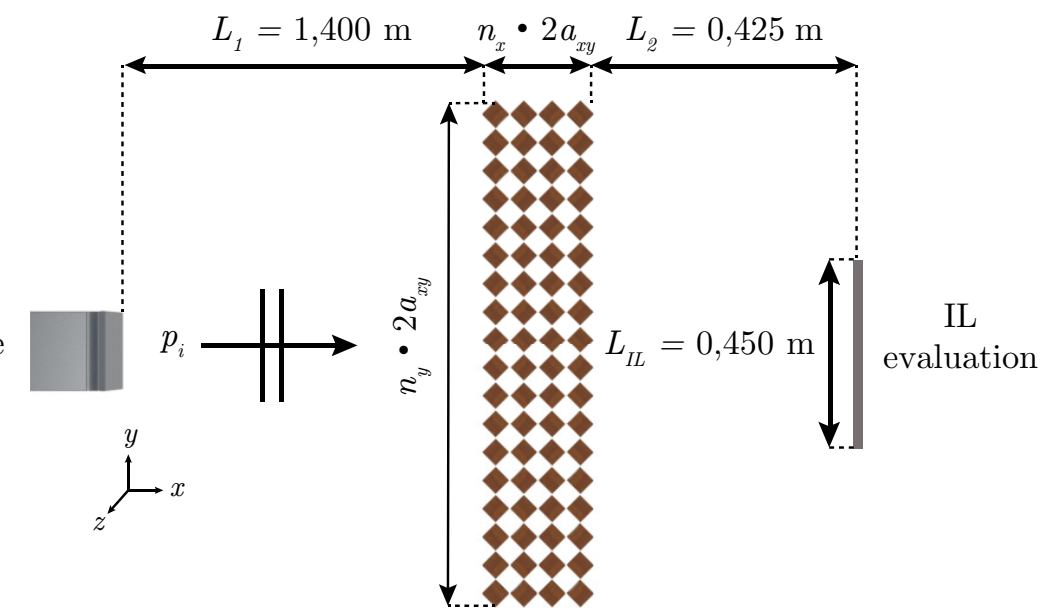

(b)

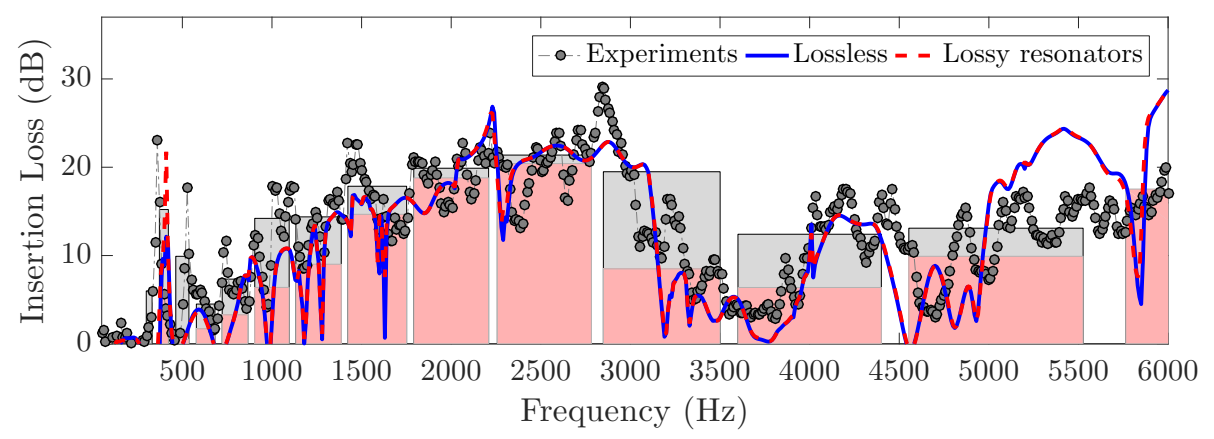

Figure 5: (a) Schematic diagram of the finite LRSC slab used for numerical simulations. (b) Comparison of the $I L$ calculated numerically, with (red dashed line) and without (blue continuous line) losses, and experimentally measured (grey circles). Bars represent $I L$ in thirds of octave for the numerical case with losses (red bars) and experiments (grey bars).

The schematic diagram of the finite LRSC slab used for the corresponding numerical calculations is shown in Fig. 5(a), where $I L$ is evaluated following the 
same exact procedure described in Section 3.3. Numerical (blue continuous and red dashed lines for the lossless and lossy cases, respectively) and experimental (gray circles) $I L$ results for a finite LRSC slab show a very good agreement, as shown in Fig. 5(b). The finite size effect of the structure strongly affects the amplitude of the IL peaks in comparison with the infinite slab, reducing the attenuation of the structure. However, the frequencies of the $I L$ peaks present the same behavior as those of the infinite slab (see Fig. 4(f)). We notice that between $3000 \mathrm{~Hz}$ and $5000 \mathrm{~Hz}$ the experimental results show better IL than the

290 analytical model. This is due to the fact that losses are not accounted out of the resonators, and in this range of frequencies the scattering an most important phenomenon. However, our numerical model still captures the trends of the experimental results.

In order to analyze the attenuation ability of the LRSC as an acoustic barrier we have also calculated $I L$ in thirds of octave. Bars in the background of Fig. 5(b) represent the corresponding experimental (grey) and numerical (red) $I L$ values. Results show that the proposed structure increases the attenuation for all the bands between 50 and $6000 \mathrm{~Hz}$. In particular, an overall IL of $16 \mathrm{~dB}$ is produced in the range of frequencies of interest, i.e., from 350 to $6000 \mathrm{~Hz}$.

\subsection{Simplified model in a real train-track configuration}

In this Section we apply a simplified 2D model of the LRSC designed in this work to a realistic case of railway rolling noise application. The geometry is shown in Fig. 6(a). The boundaries of the train and the ground (ballast, sleepers, rail, etc.) are all considered acoustically rigid while the remaining

305 boundaries are modeled adding PML layers. The spatial domain is discretized into $2.3 \times 10^{6}$ elements. The acoustic source is modeled as a point source located at the position of the train wheel.

Figure 6(b) illustrates numerical $I L$ results, which are spatially averaged along a human-sized vertical line (chosen to be $1.75 \mathrm{~m}$ high) located $1 \mathrm{~m}$ behind the LRSC. Frequency dependent results present a similar trend to the previous $3 \mathrm{D}$ analysis, but in this case the effect of the multiple virtual sources, due to the rigid boundary conditions in the domain, is observable. Three $I L$ snapshots show the sound pressure level maps at three frequencies of interest. At the HR resonance frequency, Fig. 6(c), the omnidirectional effect of the stop band is 315 represented by a strong attenuation of the acoustic field behind the structure. The effect of the ground 32 and reflections produced by the rigid boundary conditions of the walls of the train and the ground are strongly attenuated by the crystal, creating an acoustic shadow region behind the LRSC.At higher frequencies, at $f_{\text {coupling }}=1390 \mathrm{~Hz}$, Fig. 6(d) shows how the coupling of the

320 QWR attenuate the incident wave on the LRSC. Finally, at the Bragg frequency, shown in Fig. 6(e), the band gap is producing a strong attenuation in the region under analysis. 
(a)

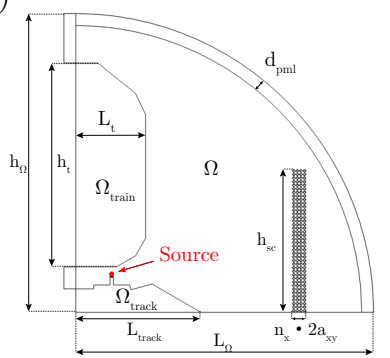

(c) $f_{H R, 2 D}=800 \mathrm{~Hz}$

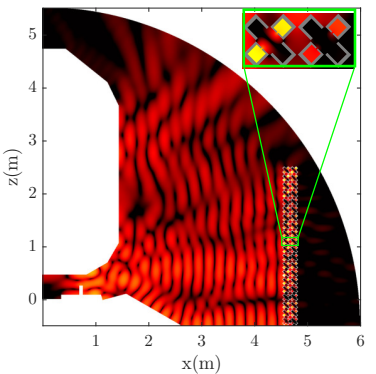

(b)

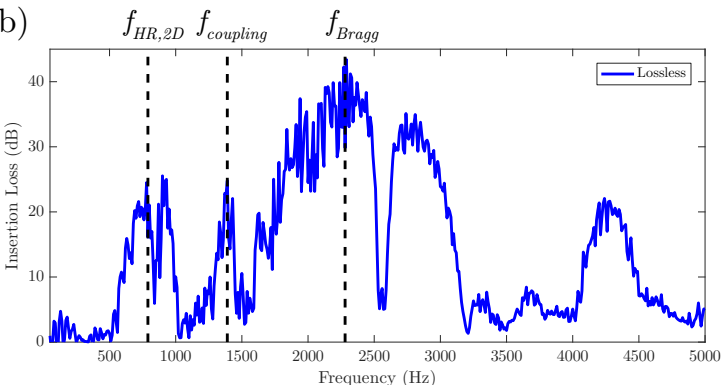

(d) $f_{\text {coupling }}=1390 \mathrm{~Hz}$

(e)
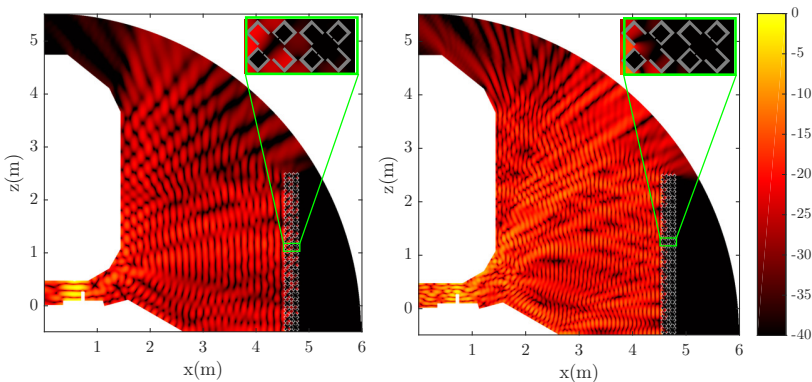

Figure 6: Simplified 2D model in a real train-track configuration. (a) Geometry of the numerical model. (b) Spatially averaged $I L$ results measured along a vertical line behind the LRSC. Acoustic pressure level (in $\mathrm{dB}$ ) in the domain at three frequencies of interest: (c) Resonance of the HR in $2 \mathrm{D}, f_{H R}=800 \mathrm{~Hz}$, (d) Coupling between the QWR, $f_{\text {coupling }}=1390 \mathrm{~Hz}$, and (e) Bragg frequency, $f_{\text {Bragg }}=2280 \mathrm{~Hz}$

\section{Conclusions}

A LRSC made of square rod scatterers with embedded HR and QWR is theoretically and experimentally analyzed in this work. The system is designed to work in the range of frequencies corresponding to various railway noise sources, in particular for the case of rolling noise. We exploit the idea of combining local resonators to generate multiple coupled resonances at low frequencies with Bragg band gaps arising from periodicity. We have considered both the effect of viscothermal losses and finite-size effects of the structure. Viscothermal losses affect the resonators efficiency at their resonances and are negligible for other frequencies. The effect of the transversal length of the structure strongly affects the $I L$ peak amplitudes. In the case analyzed in this article, a LRSC of $30 \mathrm{~cm}$ width, strong broadband attenuation is obtained: the structure exhibits a large $I L$ covering three and a half octaves with an average value of $16.8 \mathrm{~dB}$ ranging from $350 \mathrm{~Hz}$ to $5000 \mathrm{~Hz}$. These values can be greatly improved in real situations where larger structures of the same thickness can be built. We finally employ the LRSC in a simplified 2D geometry to analyze numerically the attenuation capabilities of the system in a real train-track configuration. The good efficiency of the proposed design to attenuate railway rolling noise opens new routes for designing efficient systems, with unprecedented advantages for increasing the at- 
tenuation. Moreover, low flow resistivity and allowing the light to pass through the crystal ensures suitability of the proposed structure for urban contexts. 


\section{Appendix A}

The best combination of HRs and QWRs is chosen after evaluating the dispersion relations of multiple possible $2 \mathrm{D}$ combinations. Figure 7 illustrates full and pseudo band gaps along the $\Gamma \mathrm{X}$ direction for several combinations.

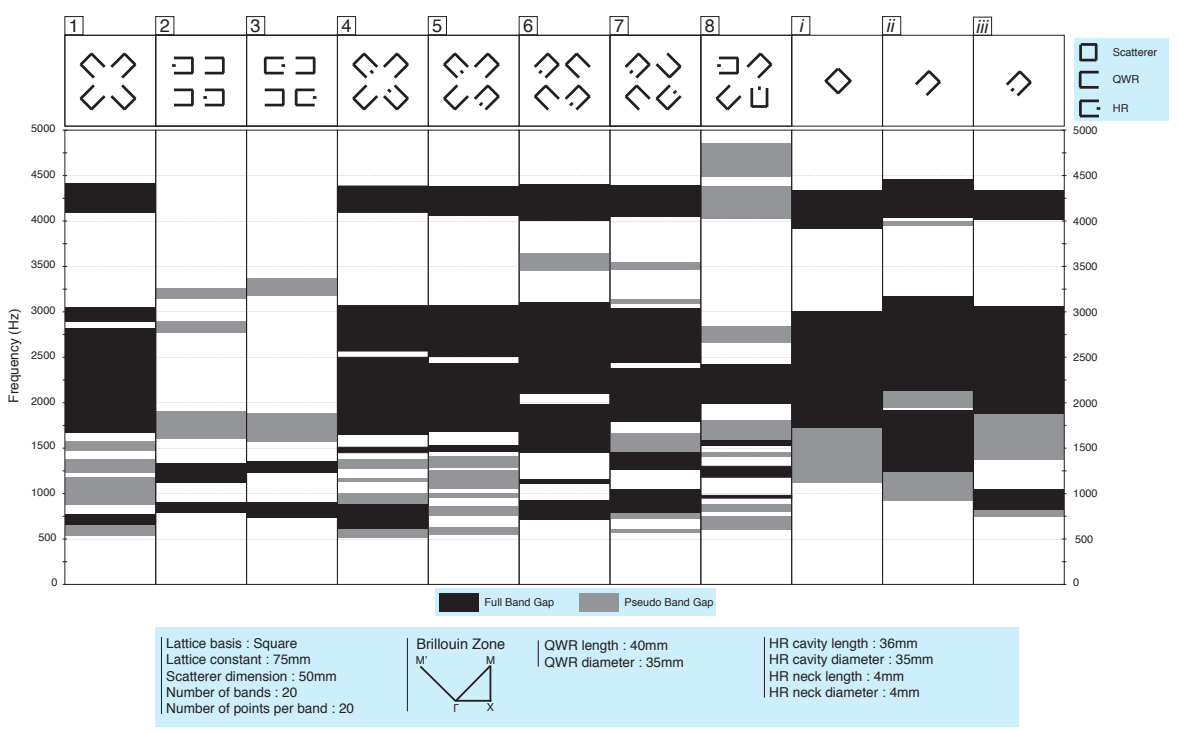

Figure 7: Preliminary studies of multiple LRSC unit cells. Pseudo band gaps ( $\Gamma \mathrm{X})$, in grey, and full band gaps, in black, for multiple coupling configurations of the LRSC. The frequency range spans from $0 \mathrm{~Hz}$ to $5000 \mathrm{~Hz}$. Columns (1) to (8) represent $2 a_{x y} \times 2 a_{x y}$ supercells, while (i), (ii) and (iii) represent $a_{x y} \times a_{x y}$ unit cells. The configuration shown in column (4) is the one employed throughout this work as it predicts the largest band gaps in the frequency range of interest.

\section{References}

[1] C. E. Bradley, Acoustic bloch wave propagation in a periodic waveguide, Tech. rep., Technical Report of Applied Research Laboratories, Report No. ARL-TR-91-19 (July), The University of Texas at Austin (1991).

[2] N. Sugimoto, T. Horioka, Dispersion characteristics of sound waves in a tunnel with an array of helmholtz resonators, J. Acoust. Soc. Am. 97 (1995) 1446.

[3] Z. Liu, X. Zhang, Y. Mao, Y. Zhu, Z. Yang, C. Chan, P.Sheng, Locally resonant sonic materials, Science 289 (2000) 1734. 
[4] N. Fang, D. Xi, J. Xu, M. Ambati, W. Srituravanich, C. Sun, X. Zhang, Ultrasonic metamaterials with negative modulus, Nat. Mater. 5 (2006) 452456.

[13] C. Lagarrigue, J. P. Groby, V. Tournat, Sustainable sonic crystal made of resonating bamboo rods, J. Acoust. Soc. Am. 133 (1) (2013) 247.

[14] A. Krynkin, O. Umnova, A. Y. B. Chong, S. Taherzadeh, K. Attenborough, Predictions and measurements of sound transmission through a periodic array of elastic shells in air, J. Acoust. Soc. Am. 128 (6) (2010) 3496-3506. arXiv:https://doi.org/10.1121/1.3506342, doi:10.1121/1.3506342. URL https://doi.org/10.1121/1.3506342

[15] F. Koussa, J. Defrance, P. Jean, P. Blanc-Benon, Acoustical efficiency of a sonic crystal assisted noise barrier, Acta Acust. United Ac. 99 (3) (2013) 399-409.

[16] S. Castiñeira-Ibáñez, V. Romero-García, J. V. Sánchez-Pérez, L. M. GarciaRaffi, Overlapping of acoustic bandgaps using fractal geometries, EPL 92 (2010) 24007. 
[17] J. Sánchez-Dehesa, V. García-Chocano, D. Torrent, F. Cervera, S. Cabrera, F. Simon, Noise control by sonic crystal barriers made of recycled materials, J. Acoust. Soc. Am. 129 (1173).

[18] V. García-Chocano, S. Cabrera, J. Sánchez-Dehesa., Broadband sound absorption by lattices of microperforated cylindical shells, Appl. Phys. Lett. 101 (18) (2012) 184101.

[19] V. García-Chocano, J. Sánchez-Dehesa, Optimum attenuation of broadband noise by sonic crystals made of recycled materials, Appl. Acoust. 74 (2013) 58 .

[20] S. Castiñeira-Ibáñez, C. Rubio, J. Sánchez-Pérez, Environmental noise control during its transmission phase to protect buildings. design model for acoustic barriers based on arrays of isolated scatterers, Build. Environ. 93 (2015) 179.

[21] A. Lardeau, J. Groby, V. Romero-García, Broadband transmission loss using the overlap of resonances in 3d sonic crystals., Crystals 6 (5) (2015) 51.

[22] C. Zwikker, C. Kosten, Sound Absorbing Materials, Elsevier Publishing Company, 1949.

[23] M. R. Stinson, The propagation of plane sound waves in narrow and wide circular tubes, and generalization to uniform tubes of arbitrary cross-

11 sectional shape, J. Acoust. Soc. Am. 89 (2) (1991) 550-558. arXiv:https: //doi.org/10.1121/1.400379, doi:10.1121/1.400379. URL https://doi.org/10.1121/1.400379

[24] L. Schwan, A. Geslain, V. Romero-García, J.-P. Groby, Complex dispersion relation of surface acoustic waves at a lossy metasurface, Appl. Phys. Lett. 110 (5) (2017) 051902. arXiv:https://doi.org/10.1063/1.4975120,

420 doi:10.1063/1.4975120 URL https://doi.org/10.1063/1.4975120

[25] L. Kinsler, A. R. Frey, A. Coppens, J. V. Sanders, Fundamentals of acoustics (4th. Ed.), John Wiley and Sonc, Inc., New York, 2000.

[26] V. Romero-García, G. Theocharis, O. Richoux, V. Pagneux, Use of complex frequency plane to design broadband and sub-wavelength absorbers, J. Acoust. Soc. Am. 139 (2016) 3395.

[27] J. Kergomard, A. Garcia, Simple discontinuities in acoustic waveguide at low frequencies: Critical analysis and formulae, J. Sound Vib. 114 (3) (1987) 465.

[28] V. Dubos, J. Kergomard, A. Khettabi, J. P. Dalmont, D. H. Keefe, C. J. Nederveen, Theory of sound propagation in a duct with a branched tube using modal decomposition, Acta Acust. United Ac. 85 (2) (1999) 153-169. 
[29] C. Goffaux, J. P. Vigneron, Theoretical study of a tunable phononic band gap system, Phys. Rev. B 64 (2001) 075118.

435 [30] N. Jiménez, W. Huang, V. Romero-García, V. Pagneux, J.-P. Groby, Ultrathin metamaterial for perfect and quasi-omnidirectional sound absorption,

1 Appl. Phys. Lett. 109 (12) (2016) 121902. arXiv:https://doi.org/10. 1063/1.4962328, doi:10.1063/1.4962328

URL https://doi.org/10.1063/1.4962328

440 [31] N. Jiménez, V. Romero-García, V. Pagneux, J.-P. Groby, Rainbow-

1 trapping absorbers: Broadband, perfect and asymmetric sound absorption by subwavelength panels for transmission problems, Sci. Rep. 7 (1) (2017) 13595. doi:10.1038/s41598-017-13706-4. URL https://doi.org/10.1038/s41598-017-13706-4

[32] V. Romero-García, J. V. Sánchez- Pérez and L. M. Garcia-Raffi, Analysis

1 of the wave propagation properties of a periodic array of rigid cylinders

1. perpendicular to a finite impedance surface, EPL, 96 (2011) 44003. doi: 10.1209/0295-5075/96/44003

URL https://doi.org/10.1209/0295-5075/96/44003 